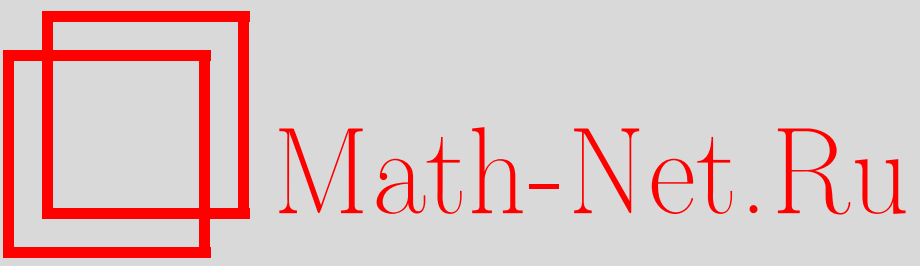

У. А. Розиков, Структуры разбиений на классы смежности группового представления дерева Кэли по нормальным делителям конечного индекса и их применения для описания периодических распределений Гиббса, ТМФ, 1997, том 112 , номер 1, 170-175

DOI: https://doi.org/10.4213/tmf1037

Использование Общероссийского математического портала Math-Net.Ru подразумевает, что вы прочитали и согласны с пользовательским соглашением

http: //www.mathnet.ru/rus/agreement

Параметры загрузки:

IP : 54.197 .130 .99

26 апреля 2023 г., $07: 47: 35$ 


\title{
У.А. Розиков*
}

\section{СТРУКТУРЫ РАЗБИЕНИЙ НА КЛАССЫ СМЕЖНОСТИ ГРУППОВОГО ПРЕДСТАВЛЕНИЯ ДЕРЕВА КЭЛИ ПО НОРМАЛЬНЫМ ДЕЛИТЕЛЯМ КОНЕЧНОГО ИНДЕКСА И ИХ ПРИМЕНЕНИЯ ДЛЯ ОПИСАНИЯ ПЕРИОДИЧЕСКИХ РАСПРЕДЕЛЕНИЙ ГИББСА}

\begin{abstract}
Описан порядок расположения элементов разбиения на классы смежности группового представления дерева Кэли по нормальным делителям конечного индекса на дереве Кэли. Для неоднородной модели Изинга доказано, что существуют три $H_{0}$-периодических распределения Гиббса $\left(H_{0}\right.$ - нормальный делитель конечного индекса).
\end{abstract}

\section{1. ВВЕДЕНИЕ}

Пусть $\Gamma^{k}=(V, L)-$ дерево Кэли порядка $k \geq 1$, где $V$ - множество вершин и $L$ - множество ребер.

ПреДЛОЖЕНИЕ 1 [1]. Существует взаимно однозначное соответствие между множеством вершин $V$ дерева Кәли порядка $k \geq 1$ и группой $G_{k+1}$ свободного произведения $k+1$ циклических групп второго порядка с образующими $a_{1}, a_{2}, \ldots, a_{k+1}$, соответственно.

Определим на $G_{k+1}$ структуру графа следуюшим образом: вершины, соответствуюшие словам $g, h \in G_{k+1}$, назовем ближайшими соседями и соединим ребром, если либо $g=h a_{i}$, либо $h=g a_{j}$ для некоторого $i$ или $j$. Нетрудно убедиться, что определенный таким образом граф̆ образует дерево Кэли порядка $k$. На $G_{k+1}$ структуру графа можно определить и по-другому, а именно, вершины, соответствуюшие словам $g, h \in G_{k+1}$, соединяются ребром, если $h=a_{i} g$, либо $g=a_{j} h$ для некоторого $i$ или $j$.

В работе [2] построены классы нормальных делителей конечного индекса группы $G_{k+1}$. Для разбиения на классы смежности группы $G_{k+1}$ относительно одной подгруппы индекса два описана структура такого разбиения, т.е. порядок расположения элементов разбиения на дереве Кэли. По этому разбиению определены периодические распределения Гиббса модели Изинга [3].

\footnotetext{
${ }^{*}$ Институт математики АН РУз, Ташкент, Республика Узбекистан
} 
В данной работе мы рассмотрим структуру разбиений дерева Кэли относительно любого нормального делителя конечного индекса и применим это разбиение для описания периодических распределений Гиббса неоднородной модели Изинга.

Неоднородная модель Изинга задается гамильтонианом [4]

$$
H(\sigma)=\sum_{\langle x, y\rangle} J_{x y} \sigma(x) \sigma(y),
$$

где суммирование ведется по всем парам ближайших соседей и $J_{x y} \in R, \sigma(x) \in\{-1,1\}$ для любых $x, y \in V$.

\section{2. СТРУКТУРЫ РАЗБИЕНИЯ ДЕРЕВА КЭЛИ}

Пусть $H_{0} \subset G_{k+1}-$ произвольный нормальный делитель индекса $n$ групшы $G_{k+1}$ (см. [2]). Ясно, что любой нормальный делитель группы $G_{k+1}$ является ядром некоторого гомоморфизма $\varphi$ группы $G_{k+1}$ в некоторую группу $G^{*}$. На множестве $G_{k+1}$ определяется следуюшее отношение эквивалентности: $x \sim y$, если $x y^{-1} \in H_{0}$.

ТЕОРема 1: 1) $x y \sim x z$ тогда и только тогда, когда $y \sim z, \quad x, y, z \in G_{k+1}$; 2) $y x \sim z x$ тогда и только тогда, когда $y \sim z, x, y, z \in G_{k+1}$.

ДокАЗАТЕЛЬСтво: 1. Необходимость. Пусть $x y \sim x z$, т.е. $x y(x z)^{-1}=$ $x y z^{-1} x^{-1} \in H_{0}$, откуда $\varphi\left(x y z^{-1} x^{-1}\right)=e \in G^{*}\left(e-\right.$ единичный элемент группы $\left.G^{*}\right)$. Поскольку $\varphi-$ гомоморфизм, то

$$
\varphi(x) \varphi\left(y z^{-1}\right) \varphi\left(x^{-1}\right)=e
$$

или $\varphi\left(y z^{-1}\right)=[\varphi(x)]^{-1}\left[\varphi\left(x^{-1}\right)\right]^{-1}=\varphi\left(x^{-1}\right)\left[\varphi\left(x^{-1}\right)\right]^{-1}=e$, т.е. $y \sim z$.

2. Достаточность. Пусть $y \sim z$, т.е.

$$
\varphi\left(y z^{-1}\right)=e
$$

Рассмотрим $\varphi\left(x y z^{-1} x^{-1}\right)$. В силу гомоморфности $\varphi$ и (3) получим $\varphi\left(x y z^{-1} x^{-1}\right)=$ $\varphi(x) \varphi\left(y z^{-1}\right) \varphi\left(x^{-1}\right)=\varphi(x) \varphi\left(x^{-1}\right)=e$, т.е. $x y \sim x z$.

Пункт 1 доказан. Пункт 2 доказывается аналогично. Теорема доказана.

СлЕДСТВИЕ 1: 1) $x a_{i} \sim x a_{j}$ тогда и только тогда, когда $a_{i} \sim a_{j}$, где $a_{i}, a_{j} \in$ $\left.\left\{e, a_{1}, \ldots, a_{k+1}\right\}, x \in G_{k+1} ; 2\right) x a_{i} \sim$ yа $a_{i}$ тогда и только тогда, когда $x \sim y$, где $a_{i} \in\left\{e, a_{1}, \ldots, a_{k+1}\right\}, x, y \in G_{k+1}$.

Обозначим через $S(x)=\left\{y \in G_{k+1}:\langle x, y\rangle\right\}$ множество ближайших соседей слова $x \in G_{k+1}$. Пусть $G_{k+1} / H_{0}=\left\{H_{0}, H_{1}, \ldots, H_{n-1}\right\}$ - фактор-группа относительно $H_{0}$. Кроме того, пусть $q_{i}(x)\left|S(x) \cap H_{i}\right|, i=\overline{0, n-1}$ и $Q(x)=\left(q_{0}(x), q_{1}(x), \ldots, q_{n-1}(x)\right)$, $x \in G_{k+1}$. Здесь $|\cdot|$ - число элементов множества.

Tеорема 2. Если $x \sim y$, mo $q_{i}(x)=q_{i}(y), \quad i=\overline{0, n-1}$.

ДокАЗАТЕЛЬСТво. Пусть $x \sim y$. Тогда в силу следствия $1 x a_{i} \sim y a_{i}$ для любого $i=\overline{0, n-1}$. Таким образом, если $S(x) \cap H_{i}=\left\{x a_{i_{1}}, x a_{i_{2}}, \ldots, x a_{i_{q_{i}(x)}}\right\}$, то $S(y) \cap H_{i}=$ $\left\{y a_{i_{1}}, y a_{i_{2}}, \ldots, y a_{i_{q_{i}(x)}}\right\}, i=\overline{0, n-1}$, т.е. $\left|S(x) \cap H_{i}\right|=\left|S(y) \cap H_{i}\right|$. Теорема доказана. 
СлеДСтвие 2. Если $x \sim y, m o Q(x)=Q(y)$.

Введем следующие обозначения: $q_{i}\left(H_{0}\right)=q_{i}(e)=\left|\left\{j: a_{j} \in H_{i}\right\}\right|, \quad Q\left(H_{0}\right)=$ $\left(q_{0}\left(H_{0}\right), \ldots, q_{n-1}\left(H_{0}\right)\right)$ и $N\left(H_{0}\right)=\left|\left\{j: q_{j}\left(H_{0}\right) \neq 0\right\}\right|$.

Теорема 3. Для любого $x \in G_{k+1}$ существует перестановка $\pi_{x}$ координат вектора $Q\left(H_{0}\right)$ такая, что

$$
\pi_{x} Q\left(H_{0}\right)=Q(x)
$$

ДОкАЗАТЕЛЬСтво. Ясно, что $S(x)=x S(e)=x\left\{a_{1}, a_{2}, \ldots, a_{k+1}\right\}=$ $\left\{x a_{1}, x a_{2}, \ldots, x a_{k+1}\right\}$. В силу следствия 1 для любого $i=\overline{0, n-1}$ сушествует единственное $j(i) \in\{0,1, \ldots, n-1\}$ такое, что $q_{i}\left(H_{0}\right)=\left|\left\{j: a_{j} \in H_{i}\right\}\right|=\mid\left\{x a_{m}: x a_{m} \in\right.$ $\left.H_{j(i)}\right\} \mid=q_{j(i)}(x)$. Положим $\pi_{x}(i)=j(i)$. Теорема доказана.

Пусть $N(x)=\left|\left\{j: q_{j}(x) \neq 0\right\}\right|$.

СлЕДСТВИЕ 3. Для любого $x \in G_{k+1}$ имеем $N(x)=N\left(H_{0}\right)$.

ДокАЗАТЕЛЬСТво следует из равенства (4).

\section{3. ОПИСАНИЕ ПЕРИОДИЧЕСКИХ РАСПРЕДЕЛЕНИЙ ГИББСА НЕОДНОРОДНОЙ МОДЕЛИ ИЗИНГА}

Пусть $A \subset V$ - конечное подмножество. Обозначим через $\Omega_{A}=\{-1,1\}^{A}$ пространство конфигураций на множестве $A$. Пусть $h_{x}$ - действительнозначная функция $x \in V$.

Рассмотрим для каждого $n>0$ меру на $\Omega_{V_{n}}$, определенную формулой

$$
\mu_{n}\left(\sigma_{n}\right)=Z_{n}^{-1} \exp \left\{-\beta \sum_{\langle x, y\rangle \in L_{n}} J_{x y} \sigma(x) \sigma(y)+\sum_{x \in W_{n}} h_{x} \sigma(x)\right\},
$$

где $\beta=1 / T$ ( $T>0$ - температура), $W_{n}=\left\{x \in V: d\left(x^{0}, x\right)=n\right\}$,

$$
L_{n}=\left\{\langle x, y\rangle \in L: x, y \in V_{n}=\bigcup_{m=0}^{n} W_{m}\right\}, \quad \sigma_{n}=\left\{\sigma(x), x \in V_{n}\right\} \in \Omega_{V_{n}}
$$

и $Z_{n}$ - нормирующий множитель.

Условие согласования для $\mu_{n}\left(\sigma_{n}\right), n \geq 1$, определяется равенством

$$
\sum_{\sigma^{(n)}} \mu_{n}\left(\sigma_{n-1}, \sigma^{(n)}\right)=\mu_{n-1}\left(\sigma_{n-1}\right)
$$

где $\sigma^{(n)}=\left\{\sigma(x), x \in W_{n}\right\}$.

Будем говорить, что $x<y$, если путь из $x^{0}$ в $y$ проходит через вершину $x$, где $x^{0}-$ фиксированная вершина. Если при этом $x$ и $y$ - соседние вершины, то $y$ называется "прямым потомком" точки $x$.

Обозначим через $\widetilde{S}$ множество "прямых потомков" точки $x[5]$. 
ТЕОРема 4 [6]. Для того чтобы меры (5) удовлетворяли условию (6), необходимо и достаточно, чтобъ для $h_{x}, x \in V$, вьполнялось равенство

$$
h_{x}=\sum_{y \in \widetilde{S}(x)} f\left(h_{y}, \theta_{x y}\right)
$$

əде $f(h, \theta)=\operatorname{arcth}(\theta \operatorname{th} h) u \theta_{x y}=\operatorname{th}\left(J_{x y} / T\right)$.

Из этой теоремы следует, что для любой совокупности $h=\left(h_{x}, x \in V\right)$ существует и притом единственное распределение Гиббса $\mu$, которое удовлетворяет равенству $\mu\left(\sigma_{n}\right)=\mu_{n}\left(\sigma_{n}\right)$.

Пусть $H_{0} \subset G_{k+1}-$ нормальный делитель индекса $n$. Обозначим $x_{\downarrow}=S(x) \backslash \widetilde{S}(x)$. По построению ясно, что класс принадлежности $x_{\downarrow}$ зависит от места нахождения слова $x$ на дереве. Поэтому $\widetilde{S}(x)$ также зависит от места нахождения слова $x$. Чтобы получить $H_{0}$ - периодические распределения Гиббса, предположим, что $h_{x}=h_{i}$, если $x \in H_{i} \in$ $G_{k+1} / H_{0}, i=\overline{0, n-1}$.

Пусть $N\left(H_{0}\right)=\left|\left\{q_{i_{1}}\left(H_{0}\right), \ldots, q_{i_{N\left(H_{0}\right)}}\left(H_{0}\right)\right\}\right|, \theta_{x y}=\theta_{i j}$, если $x \in H_{i}, y \in H_{j}$. В силу теорем 2-4 имеем

$$
h_{m}=\sum_{j=1}^{N\left(H_{0}\right)} q_{i_{j}}\left(H_{0}\right) f\left(h_{\pi_{m}\left(i_{j}\right)}, \theta_{m \pi_{m}\left(i_{j}\right)}\right)-f\left(h_{\pi_{m}\left(i_{j_{0}}\right)}, \theta_{m \pi_{m}\left(i_{j_{0}}\right)}\right) \text {, }
$$

где $m=\overline{0, n-1}, j_{0}=\overline{1, N\left(H_{0}\right)}$ и $\pi_{m}$ определяется по теореме 3 .

ТЕОрема 5. Для того чтобъ вектор $h^{*}=\left(h_{0}^{*}, h_{1}^{*}, \ldots, h_{n-1}^{*}\right)$ являлся решением системы (8), необходимо, чтобы для любого $m=\overline{0, n-1}$ выполнялись равенства

$$
f\left(h_{\pi_{m}\left(i_{1}\right)}^{*}, \theta_{m \pi_{m}\left(i_{1}\right)}\right)=f\left(h_{\pi_{m}\left(i_{2}\right)}^{*}, \theta_{m \pi_{m}\left(i_{2}\right)}\right)=\cdots=f\left(h_{\pi_{m}\left(i_{N}\right)}^{*}, \theta_{m \pi_{m}\left(i_{N}\right)}\right) .
$$

ДоКАЗАТЕЛЬСТво следует из (8).

Пусть даны числа $c_{1}, c_{2}, \ldots, c_{n-1} \in R$. Решение системы (8) представим в виде

$$
h_{i}=f\left(h_{0}, c_{i}\right), \quad i=\overline{1, n-1} .
$$

Лемма 1. Имеет место соотношение $f(f(h, \theta), \delta)=f(h, \theta \delta)$, где $h, \theta, \delta \in R$.

ДокАЗАТЕЛЬСТво. По определению функции $f$ имеем $f(f(h, \theta), \delta)=$ $\operatorname{arcth}(\delta \operatorname{th}(\operatorname{arcth}(\theta \operatorname{th} h)))=\operatorname{arcth}(\delta \theta \operatorname{th} h)=f(h, \theta \delta)$. Лемма доказана.

Подставляя (10) в (8), в соответствии с леммой 1 получим

$$
\begin{aligned}
h_{m}= & \sum_{j=1}^{N\left(H_{0}\right)} q_{i_{j}}\left(H_{0}\right) f\left(h_{0}, c_{\pi_{m}\left(i_{j}\right)}, \theta_{m \pi_{m}\left(i_{j}\right)}\right)- \\
& -f\left(h_{0}, c_{\pi_{m}\left(i_{j_{0}}\right)}, \theta_{m \pi_{m}\left(i_{j_{0}}\right)}\right), \quad m=\overline{0, n-1}, \quad j_{0}=\overline{1, N\left(H_{0}\right)} .
\end{aligned}
$$


При $m=0$ имеем

$$
h_{0}=\sum_{j=1}^{N\left(H_{0}\right)} q_{i_{j}}\left(H_{0}\right) f\left(h_{0}, c_{i_{j}} \theta_{0 i_{j}}\right)-f\left(h_{0}, c_{i_{j_{0}}} \theta_{0 i_{j_{0}}}\right), \quad j_{0}=\overline{1, N\left(H_{0}\right)} .
$$

Подставляя (10) в (9), опять в силу леммы 1 находим

$$
c_{\pi_{m}}\left(i_{1}\right) \theta_{m \pi_{m}\left(i_{1}\right)}=c_{\pi_{m}\left(i_{2}\right)} \theta_{m \pi_{m}\left(i_{2}\right)}=\cdots=c_{\pi_{m}\left(i_{N\left(H_{0}\right)}\right)} \theta_{m \pi_{m}\left(i_{N\left(H_{0}\right)}\right)}, \quad m=\overline{0, n-1} .
$$

Решая (11) относительно $h_{0}$, видим, что если

$$
\sum_{j=1}^{N\left(H_{0}\right)} q_{i_{j}}\left(H_{0}\right) c_{i_{j}} \theta_{0 i_{j}}-c_{i_{j_{0}}} \theta_{0 i_{j_{0}}}>1
$$

то уравнение (11) имеет три решения: $-h_{0}^{*}, 0, h_{0}^{*}$. Подставляя $h_{0}= \pm h_{0}^{*}$ в $(10)$ и $\left(10^{\prime}\right)$ и сравнивая правые части этих равенств, получаем

$$
\begin{gathered}
f\left( \pm h_{0}^{*}, c_{m}\right)=\sum_{j=1}^{N\left(H_{0}\right)} q_{i_{j}}\left(H_{0}\right) f\left( \pm h_{0}^{*}, c_{\pi_{m}\left(i_{j}\right)}, \theta_{m \pi_{m}\left(i_{j}\right)}\right)-f\left( \pm h_{0}^{*}, c_{\pi_{m}\left(i_{j_{0}}\right)}, \theta_{m \pi_{m}\left(i_{j_{0}}\right)}\right) \\
m=\overline{0, n-1}, \quad j_{0}=\overline{1, N\left(H_{0}\right)} .
\end{gathered}
$$

Таким образом мы доказали следуюшую теорему.

Теорема 6. Если $c_{1}, c_{2}, \ldots, c_{n-1}$ заданы и параметры $\theta_{i j}, i, j=\overline{0, n-1}$, удовлетворяют условиям (12), (12') и (13), то система (8) имеет три решения: $h^{*}=(0,0, \ldots, 0) u \pm h^{*}=\left( \pm h_{0}^{*}, h_{1}^{*}, h_{2}^{*}, \ldots, h_{n-1}^{*}\right)$, дде $h_{i}^{*}=f\left( \pm h_{0}^{*}, c_{i}\right), \quad h_{0}^{*} \neq 0$, $i=\overline{1, n-1}$.

Верна теорема и обратная к теореме 6.

Теорема 7. Если $h^{*}=\left(h_{0}^{*}, h_{1}^{*}, \ldots, h_{n-1}^{*}\right)$ есть решение системы (8), то существуют $c_{1}, c_{2}, \ldots, c_{n-1} \in R$ такие, что

$$
h_{i}^{*}=f\left(h_{0}^{*}, c_{i}\right), \quad i=\overline{1, n-1} .
$$

ДокАЗАтЕльство. Достаточно предположить, что $c_{i}=\frac{\operatorname{th} h_{i}^{*}}{\operatorname{th} h_{0}^{*}}$, если $h_{0}^{*} \neq 0$. Если $h_{0}^{*}=0$, тогда $c_{i}-$ произвольное число. Теорема доказана.

Из приведенных результатов вытекает наше основное утверждение.

Tеорема 8. Существует непустое подмножсество множества параметров $\left(\theta_{i j}, \quad i, j=\overline{0, n-1}\right)$, для каждой точки которого имеются три $H_{0}$-периодические распределения Гиббса, соответствующие решениям системь (8). 


\section{Список литературы}

[1] Н. Н. Ганиходжсаев. ДАН РУз. 1994. № 5. С. 3-6.

[2] Н. Н. Ганиходжаев, У. А. Розиков. ТМФ. 1997. Т. 111. № 1. С. 109-117.

[3] Н. Н. Ганиходжаев, У. А. Розиков. УЗМЖ. 1995. № 4. С. 8-19.

[4] Я. Г. Синай. Теория фазовых переходов. М.: Наука, 1980.

[5] P. M. Bleher. Commun. Math. Phys. 1990. V. 128. P. 411-419.

[6] S. Katsura, M. Takizawa. Progr. Theor. Phys. 1974. V. 51. P. 82-98.

Поступила в редакцию 5.VIII.1996 г., после доработки 16.I.1997 г.

\section{U. A. Rozikov}

\section{STRUCTURES OF PARTITION OF THE GROUP REPRESENTATION OF THE CAYLEY TREE INTO ADJACENT CLASSES BY FINITE INDEX NORMAL SUBGROUPS AND THEIR APPLICATION FOR DISCRIPTION OF PERIODIC GIBBS DISTRIBUTIONS}

The disposition order of partition elements into adjacent classes of the group representation of the Cayley tree on its finite index normal subgroups is described. For the inhomogeneous Ising model it is proved that there exist three $H_{0}$-periodic Gibbs distributions, where $H_{0}$ is a normal subgroup of finite index. 